\title{
A Virtual Community Design for Home-based Chronic Disease Healthcare
}

\author{
Yan $\mathrm{Hu}^{1}$, Guohua Bai ${ }^{1}$, Jenny Lundberg ${ }^{2}$ and Sara Eriksén ${ }^{1}$ \\ ${ }^{1}$ Dept. of Creative Technologies, Blekinge Institute of Technology, 37179, Karlskrona, Sweden \\ \{yan.hu, guohua.bai, sara.eriksen\}@bth.se \\ ${ }^{2}$ Dept. of Media Technology, Linnaeus University, 35195, Vaxjo, Sweden \\ jenny. lundbergelnu. se
}

\begin{abstract}
The internet based social network has been applied to serve many social functions, such as democratic decision making, knowledge sharing, education, and healthcare. In this paper, we provide a prototype of virtual community designed for home-based chronic diseases healthcare. We studied the concept "community" from the activity theory model in order to design the prototype with a solid theoretical base. Then we conducted a questionnaire from healthcare recipients and interviewed healthcare providers to gather the requirements for the design of the community. With some user stories we described the requirements as use cases for our design and a conceptual prototype is built based on the requirements. This virtual community servers as a shared platform for all the stakeholders who are engaged in the healthcare activity. With this shared community platform, the interoperability problems of current healthcare systems can be moderated.
\end{abstract}

Keywords: virtual community, self-management, home-based chronic disease healthcare, requirements, activity theory

\section{$1 \quad$ Introduction}

Chronic diseases are increasingly becoming a main factor influencing human health and wellbeing all over the world. According to the World Health Organization (WHO) [1], chronic diseases represent $60 \%$ of all deaths in the world, and are thus the leading cause of mortality. Chronic diseases last for a long time, and can hardly be cured [2], therefore how to provide preventive and monitoring healthcare becomes a worldwide goal. Since patients suffering from chronic diseases have to be monitored from time to time, it leads to high cost, and becomes time consuming and inconvenient for the patients. This time-to-time monitoring limits the patients' daily activities and is especially inconvenient for aged people. Since mostly chronic diseases do not need urgent medical diagnosis and treatment [3], we suggest moving the front desk of chronic diseases healthcare from hospital-based to home-based care. This shift may save a lot of patient time and medical resources, and provide a convenient environment for continued living at home despite an increasing need of monitoring of health.

adfa, p. 1, 2011.

(C) Springer-Verlag Berlin Heidelberg 2011 
Thanks to the development of information and communication technology (ICT), the above suggested home-based healthcare is now becoming highly recommended [3]. Many physiological signals can be measured by individuals in their living environments during daily activities [4]. This paper will propose one important service function for such a home-based solution, namely virtual community based webservices. With this virtual community, people with chronic diseases can quickly and directly communicate with each other (supporting peer-to-peer learning), and also with healthcare providers and family members concerning their ongoing situation. With various needed services based on this virtual community, patients can have an independent living at home with improved life quality.

This paper will start by discussing the understanding of 'community' based on social-psychological activity theory and interpreted in the concrete context of homebased healthcare for chronic diseases. In the second part, a requirement elicitation is carried out based on a survey among potential users (55+ with chronic diseases). In the third part we analyze the collected 27 answers we got concerning the most needed requirements for a virtual community. Based on the defined requirements, in the fourth part we present a prototype we have developed to demonstrate how to design a web-based virtual community to integrate those identified needs for the targeted users, especially elderly users. Finally, we discuss some weaknesses and limitations with the proposed solution, and conclude with conclusions.

\section{$2 \quad$ What is a virtual community?}

A community is broadly defined as "a group or network of persons who are connected (objectively) to each other by relatively durable social relations that extend beyond immediate genealogical ties, and who mutually define that relationship (subjectively) as important to their social identity and social practice."[5]. Since the introduction of the now widely used Internet, the concept of community today has less geographical limitation, as people can now gather virtually in an online community and share common interests regardless of physical location. This kind of community, with far less constraints due to genealogical distance and far more oriented to shared interests and objectives, is what we call a virtual community. A virtual community is a social network that people interact with through specific social media, of which there are many good examples, such as Facebook and Twitter. This virtual community allows people to communicate with each other about their common interests without considering geographical distance.

Based on the model of Activity Theory (Fig. 1), a community is generally defined as a group of people who are engaged in conducting activities to approach a shared goal or outcome. In relation to service oriented activity where the object is the recipients of the conducted service activity (not as a materialized object), the members of this community can be divided into three groups: 1) people who are actively conducting the service activities, here called a subject; 2) people who are acted on by the subject, here called an object; and 3) people who are not directly acting or being acted on, but who are associated with the ongoing activity. 
The model in Fig. 1 visualizes the components that compose an activity and interactions in an activity. According to the model, an activity is always conducted by one or several goal-oriented actors, labeled as a subject in the model. The subject is always directed towards or acting on an object in order to produce an outcome. The community in this model is the collective of the above three groups of people involved in conducting the activity. Rules are various regulations for how to conduct activities and serve as mediating function within the community if any disagreement appears in conducting the activity. Tools are artifacts (physical, conceptual, or signs) used by the subject in the process of acting in order to produce the outcome. Division of labor specifies the responsibility within the community, i.e., which parts of the object's needs should be implemented by which members of the community.

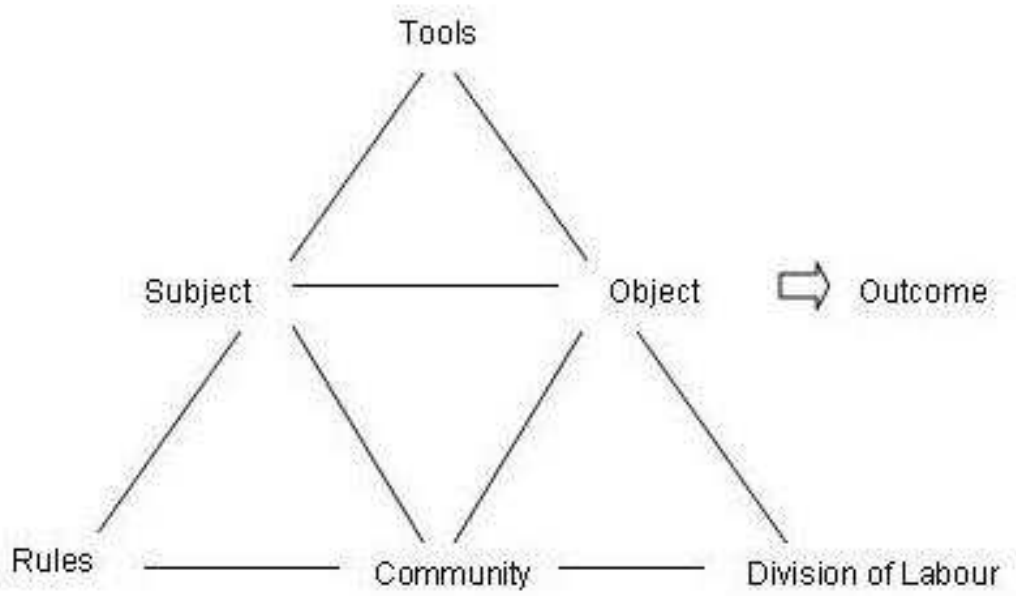

Fig. 1. Engeström's Activity Model [6]

To apply the above concept of community in the activity context of home-based healthcare, we will interpret the model in concrete terms related to the context. The outcome or goal of the activity is to improve the healthcare recipient's health. The subject is the healthcare providers such as doctors, nurses, family members. The object is the healthcare recipients, in this case they are people who are suffering from chronic diseases. Tools are all kinds of artifacts, which are used to support the activity, physical and non-physical, such as EHRs, Internet, healthcare monitor devices, as well as treatment-related knowledge and methods. The rules regulate the actions of the actors, and consist of for example healthcare laws, privacy and security policies and so on. Division of labor determines the responsibilities of different healthcare providers and healthcare recipients themselves. In this activity model, besides subject (actors) and object (recipients), the community includes also other healthcare actors, such as family members of the care recipients, other healthcare recipients who share the same interest, and research institutes that are associated with the results of the activity. 
In home-based healthcare, self-management is highly encouraged, and in this selfmanagement the healthcare recipients become the subject (actor) and the object (being self-acted) simultaneously. The healthcare recipients can also start acting in relation to the healthcare providers, for example if the patient alerts his/her nurse about newly recorded abnormal blood pressure for online consultancy. In this case the role of subject in traditional hospital care is now becoming an object in home-based healthcare. We call this property subject-object mutuality [7]. The design of the virtual community must consider different roles of actors with their expected functions and tools. In the next section, we will focus first on the healthcare recipients' perspective in the activity context of home-based healthcare.

\section{Requirements collection of community}

\subsection{Requirements from healthcare recipients}

A good virtual community should attract people with the same interests into a meeting place, but more importantly, the virtual community should in this case provide healthcare tools and services. Patient-centered self-management is the main trend for home-based chronic disease healthcare, and the virtual community in this case should first focus on the needs of the targeted patients. There are already some commercial or research-based solutions for supporting self-management. For instance, HealthVault [8], launched by Microsoft, is a web-based PHR system for storing and managing health information. A lot of specific third-party applications, such as blood pressure management tools and medical image viewers, as well as hundreds of devices such as blood glucose meters and blood pressure monitors, can cooperate with this platform to record health data.

In order to meet most users' needs when designing the online virtual community, we begin with requirements specification. There are two types of requirements in website design and software engineering, functional requirements and non-functional requirements [9]. As we mentioned above, in home-based healthcare, patients are at the center of the healthcare, as well as the main users. So we selected online questionnaires to be answered by the potential healthcare recipients as the key data-gathering technique.

Questionnaires include a series of questions designed to elicit specific information from the users. Well-designed questionnaires are a good way to get answers to specific questions from a group of people, especially for people whom it is not feasible to visit individually and interview [9]. In this case, the questions are mostly designed for functional requirements as seen from the healthcare recipients' perspective. Due to the time and resources limitation, for our questionnaires, the questions are published on surveymonkey.com as the electronic form rather than a paper form. The respondents are selected from within an age group consisting of people above 55 years of age and living with one or more chronic diseases. We send out the questionnaires through social network websites, for example, social groups of some specific chronic diseases ex. Diabetes from patientslikeme and facebook. The questionnaire contains 10 questions, and includes both simple Yes or No questions, questions allowing the respondent to choose from a set of pre-supplied answers, and open comment questions. In the 
end, 25 valid responses out of 27 are gathered. Most of the respondents are from US (8) and Sweden (12). We consider that the geographical difference may effect some results, so the questions are designed to reduce this influence as possible. From the collected answers, we find that the influence is very low.

\section{Problems in current healthcare}

The first open question is about the problems people face concerning their current healthcare. Almost all the respondents mentioned that the long time they spend waiting for doctors' appointments make them uncomfortable. "The resource is limited. It means I need to spend more time waiting for the healthcare service. And since I am waiting for the doctor, I really also need suggestions to keep my body stable, not get worse." "Really long queue every time when I visit some hospitals". In some countries like Sweden, the shortage of healthcare providers leads to long waiting lines for visiting primary care as well as hospitals. Sometimes the patients have to wait so long that they lose the best opportunity for diagnoses and treatments in relation to curing or sustainably managing their diseases. Another big problem the respondents highlighted is lack of information sharing among different healthcare providers. When they come to the new healthcare organizations, their historical healthcare records are difficult to find. "When I went to another hospital which I never visited before, the doctor didn't know my health history, if I am hypersensitive to some drugs, he didn't know, so this may cause some healthcare problems. If I need to have a new scratch test taken to determine what I am hypersensitive to, it wastes time and resources." "There is no shared information among the hospitals. It cannot help the care providers to communicate to each other." This causes overlap check-ups for the healthcare recipients, which is a waste of time and resources. Besides the above two quotes, some respondents also point out that the location of the healthcare center is far away from their home, and that it is inconvenient for them to go to healthcare centers frequently.

\section{Views of online healthcare community}

When asked about the time spent on Internet per day, $60 \%$ of the respondents spend more than 6 hours on-line, see Fig. 2, which means Internet has already become an essential part of their daily lives. Because of the high rate of Internet usage, the online healthcare community is acceptable. All the respondents say they would like to have an online healthcare community, where they could chat with all stakeholders related to their healthcare through one platform. The answers concerning which people they want to chat with through the online community show the following: doctors $(96 \%)$, other healthcare providers $(84 \%)$, other people with same symptoms $(72 \%)$, family members $(68 \%)$ and some healthcare research institutions $(64 \%)$. 
Hours spend on Internet per day

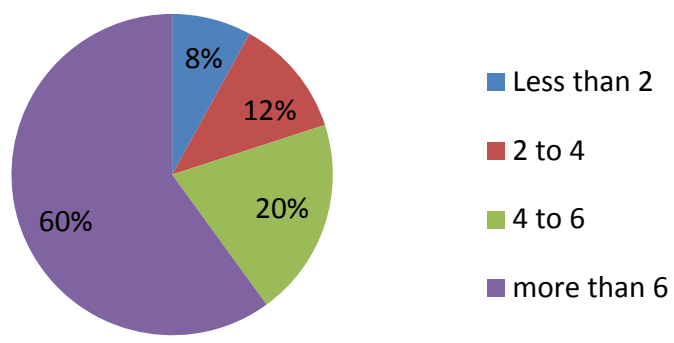

Fig. 2. Hours spent on Internet per day

\section{Online community functions design}

In Questions 5 to 8, we ask about some specific functions of the online healthcare community. The results are shown in Fig.3. From the figure, we can see that all functions we plan to develop are supported by most of the respondents. Question 10 is an open question about other functions they would like to have. Online simple diagnose was one of the most desirable functions. Free seminars and lectures about healthcare is another suggestion by most of the respondents. "Simple medical suggestions and brief medical diagnosis", "Diagnosis, chatting, forum, online seminar" "diagnosis online" and "Healthcare lecture" were mentioned most. In addition, fast contact and response, risk prediction, decision support systems and information about nearby healthcare centers were also suggested. In Question 9, the respondents were asked which features are important for them as the users of online community. Availability $(100 \%)$, usability (88\%), security ( $88 \%)$ and privacy $(84 \%)$ all got very high support.

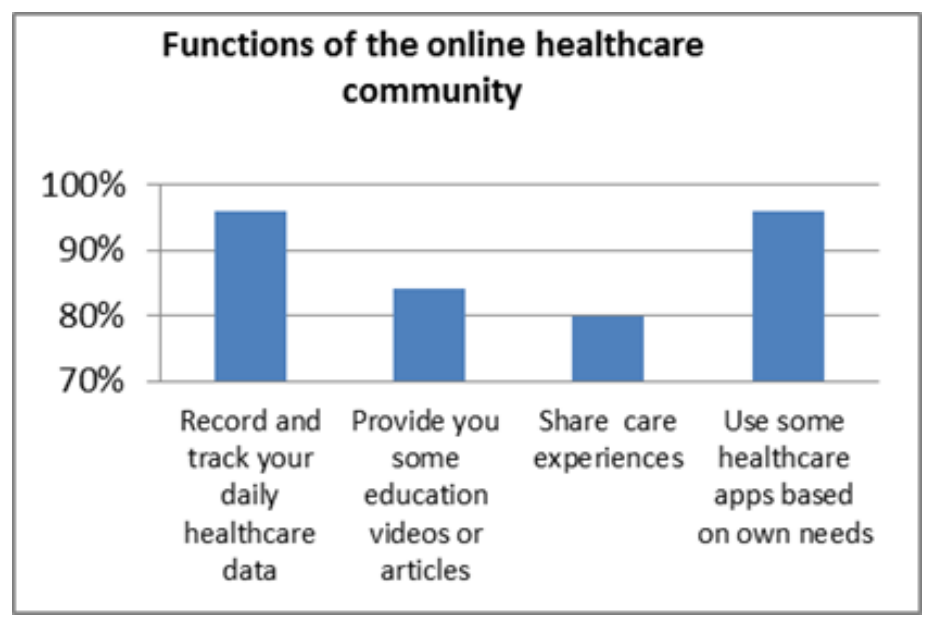

Fig. 3. Functions of the online healthcare community 


\subsection{Requirements from healthcare providers}

One of the aims of building up this online care community is to encourage the care recipients to be the main driving forces of their home-based healthcare. Still, the virtual community will only develop in a sustainable way if the tools provided support and meet the needs of all the aimed-for user groups. The requirements of healthcare providers are gathered mainly based on interviews and informal discussions with doctors, nurses and homecare providers.

In the current Swedish healthcare system, most nurses are working daily with 5 to 10 different IT systems, and only a few of these systems share data with each other. Sometimes they just record the same simple data repetitively in different systems due to this lack of compatibility between systems, which is perceived as a waste of time and in some way decreases the work enthusiasm. There is a national electronic service called National Patient Overview (Nationell Patientöversikt - NPÖ), which enables healthcare providers to share patient healthcare records with other healthcare providers through computer networks. The purposes of NPÖ are to facilitate cooperation among different healthcare providers in Sweden, as well as giving healthcare recipients access to their own healthcare information [10]. NPÖ assists cooperation among different healthcare providers at the national level. However, it is predominantly a solution for hospital-based healthcare, as the direct beneficiary of this service is the healthcare providers. Even for the healthcare providers, only doctors have full access to the records. When shifting to home-based chronic disease care, NPÖ can provide few access rights for healthcare recipients. Also, as home-based healthcare involves other parties like family members and other patients with the same symptoms, how to share data with these parties is another challenge. In addition, NPÖ is only used for sharing healthcare information in Sweden; when people go abroad, it is quite difficult for them to access their healthcare data. In Europe, there are some projects aim to provide cross-border eHealth services by exchange patients' summaries between healthcare institutions such as epSOS [11], however, most of them are still in the test phases.

The basic way of communicating with care recipients now is still face-to-face. The emergence of the national digital healthcare portal 1177 [12] helps most healthcare recipients to communicate with their providers without face-to-face visiting, but there are still several issues it cannot cover. For example, it cannot provide medical suggestions because of different care duties, and communication is lagging in some cases due to technical reasons and wrong contact responsible line. Through the minavårdkontakter contact provided by 1177 , care recipients can send some message to their providers, but the average response time is around 5 days. And from the care providers' view, a telephone call is better than sending an online message or email, because it is difficult to understand the text written by the care recipients.

For the management of chronic diseases such as diabetes, the normal way now is that the healthcare recipients go to the hospital, and are checked and given advice by nurses first. The nurses will teach the patients how to self-record every day's blood sugar values. Then when the patients go back home, they record the data as instructed and send their values via SMS to the nurse. There is an IT management system in 
hospitals for the nurses, but it does not share any information to neither other care providers nor care recipients.

\section{$4 \quad$ Main functional requirements}

In this section, we list the simple functional requirements of the online virtual community as documented through our questionnaires. Table 1 is the requirements list of the online community design. The traditional way of documenting requirements consists of contract style requirement lists [13]. This provides a high level requirements description for a large system. However such requirements descriptions usually cover hundreds of pages and are not reader-friendly. For our community design, we use the user stories style to describe the functional requirements in order to support easy and comprehensive understanding and quick changes. A user story is used with agile software development methodologies. It contains one or more sentences in everyday language to describe what an end user wants to do or needs as part of the functions [14].

Table 1. Requirements List

\begin{tabular}{ll}
\hline User types & \multicolumn{1}{c}{ Descriptions } \\
\hline All users & As a user, I want to register an account on the online healthcare \\
community and choose my user type based on different roles. \\
As a user, when I log in to my account, the information page \\
will be displayed automatically according to my user type. \\
As a user, I want to have an online chat with my contact persons \\
such as care providers, care recipients, family members and so \\
on. \\
As a user, I want to have alarms to remind me of my care activi- \\
ties. \\
As a user, I want to have the all contract persons' address book \\
with detailed contact information. \\
As a user, I want to share some useful healthcare information \\
with my contact persons. \\
As a care recipient, I want to contact my care providers directly \\
through the online community for simple diagnose and care \\
suggestions. \\
As a care recipient, I want to have some videos for providing \\
healthcare education. \\
As a care recipient, I want to record my daily health data myself \\
in the community and share it with people whom I want to share \\
with. \\
As a care recipient, I want to have some tools to trace and check \\
my care data, as well as to print it out when necessary. \\
As a care recipient, I want to share my care experiences with \\
people who have the same symptoms as I do.
\end{tabular}


As a care recipient, I want to book a doctor's appointment online.

As a care recipient, I want to integrate some healthcare relevant apps in the community based on my individual needs.

As a care recipient, I want to have the address and open hours information of the nearest healthcare centers

Care providers As a care provider, I want to share some care information with other providers.

As a care provider, I want to have some online seminars with other providers and my care recipients.

As a care provider, I want to update the care histories of my recipients to the online community.

As a care provider, I want to have some decision support systems to help me for providing diagnoses and treatments.

As a care provider, I want to have a list of every day's work.

\section{$5 \quad$ Non-functional requirement issues}

A non-functional requirement is a requirement used to measure the operation of a system, rather than specific behaviors [15]. In software systems, non-functional requirements are also considered as quality attributes. For our community design, usability, security and privacy, as well as availability are main issues of non-functional requirements [16].

Usability: Usability is defined as "the extent to which a product can be used by specified users to achieve specified goals with effectiveness, efficiency, and satisfaction in a specified context of use." [17]. According to [18] usability can be measured by five variables which are defined as learnability, efficiency, memorability, errors, and users' satisfaction. The main users of this online community are elderly people with chronic diseases, thus the designed services in the virtual community should be extremely simple to use and easy to learn. As design guidelines, we follow the "Ten usability heuristics [19]", especially principles about knowing the users' needs, special visibility for elderly, consistency with elderly's daily language, and helping them recognize previous experience (reducing recall problem for elderly).

Privacy: The patient health information includes personal information, details of medical history, symptoms, treatments, associated diseases or even the family health history. It is important to ensure that only the patients can authorize exactly who can view the shared health information and for what purposes [20]. All the information generated by a patient is not at the same sensitive level. Data segmentation may provide a method to protect specific sections of health information while giving choices to patients, and abiding requirements of legislation. Technical considerations and definition of sensitive information have to be addressed when segmenting data [21].

Security: Security is assurance that only authorized persons or entities can gain access to patients' data. Employees' illegitimate access and theft is one of the most frequent reasons of data leakage [20], as well as innocent disclosure because of system 
problems. Another issue is unauthorized access and malicious attacks from outside. So the encryption, identification and access control of patient health data are not optional for the online community development [20].

Availability: As an online healthcare community, being available $24 / 7$ is very important, because some functions, such as monitoring, tracking, alarming are critical for users' lives. Availability means also that the authorized users should be able to access the community from anywhere. More and more people, even elderly with chronic diseases are traveling worldwide, and the healthcare community must be mobile in the sense that it must allow them access from anywhere in the world, just like when they are in their own home.

\section{Prototype design}

With the requirements specified above, we will demonstrate a simple prototype in the following. We adopt a horizontal prototyping strategy, which means that the prototype should cover most parts of the required users' functions, without implementation of details[22]. Since it is a user-centered online community prototype, we try to keep it simple to use and easy to learn. The home page of the community is shown in Fig. 4, which is a very simple site for sign-up and sign-in with some guidance. In our prototype, the roles of users are divided into three types: healthcare recipient, healthcare provider and others like family members and researchers. The user type is selected by the users when they register in the system. After successfully registering, the information related to the role in the community will be displayed based on the user types. The role of the users decides the information and activities they can have access to in the online community.

When the user has finished registration and logged in to his or her account using a user name and password, the system will automatically direct the user to his or her sites according to the specified user role. As shown in Fig. 5, Anna Nilsson is a healthcare recipient, and the profile site has her basic information and the information relevant to her, such as her healthcare community, tools and rules that apply to her in her role. The contacts list in the middle has detailed information about all her care providers and other people relevant to her healthcare. In the right-bottom part, she can note her main symptoms so that people who have the same conditions can easily find her and they can share care experiences with each other. Fig. 6 is the profile page of a healthcare provider, Maria Karlsson; similar to the recipient's page, it contains her basic information and contacts list. In the right-bottom part, the tasks she has to do today are listed as a reminder. 


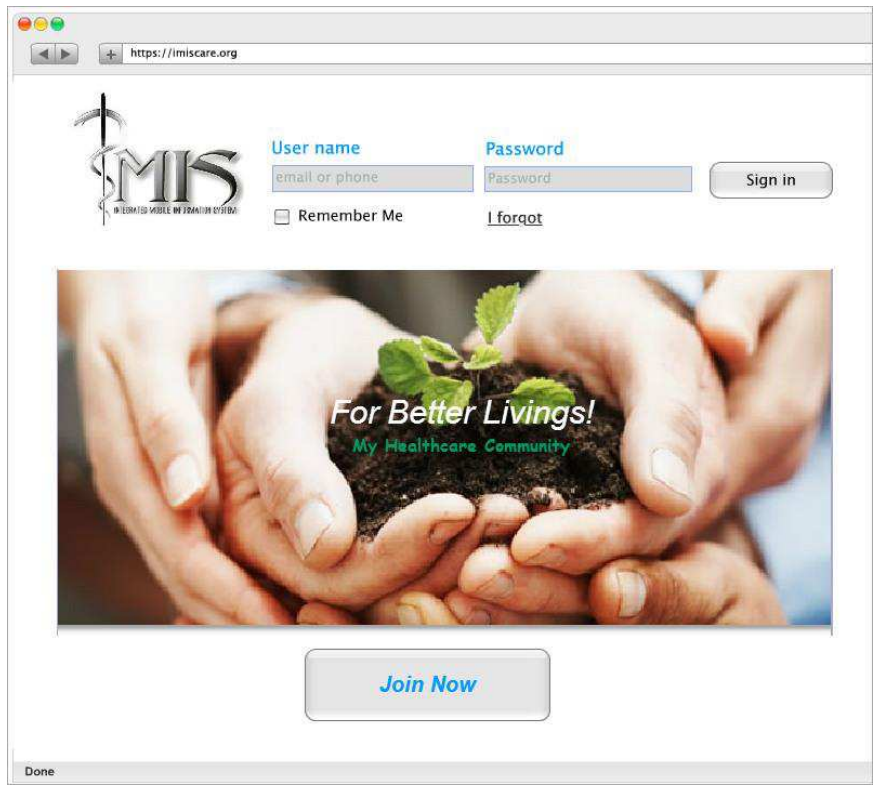

Fig. 4. Home page of the community

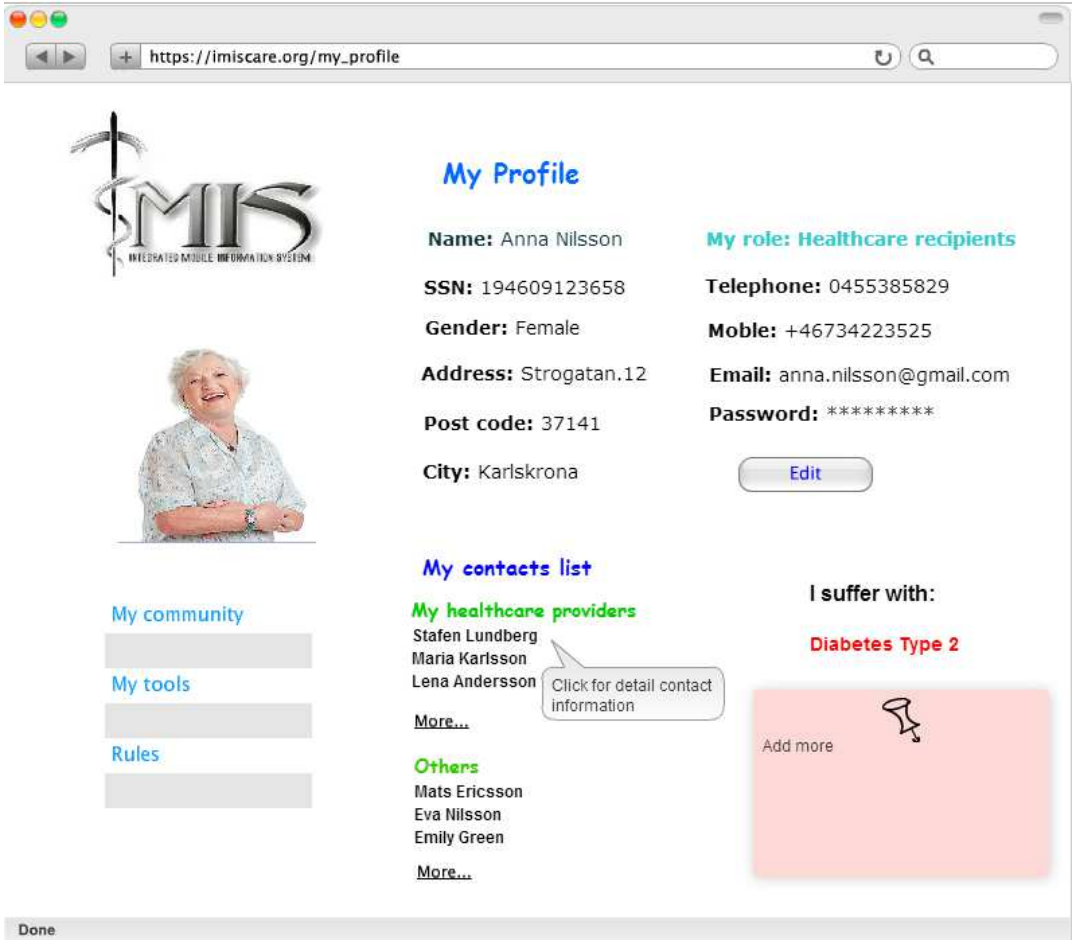

Fig. 5. Healthcare recipients' profile 


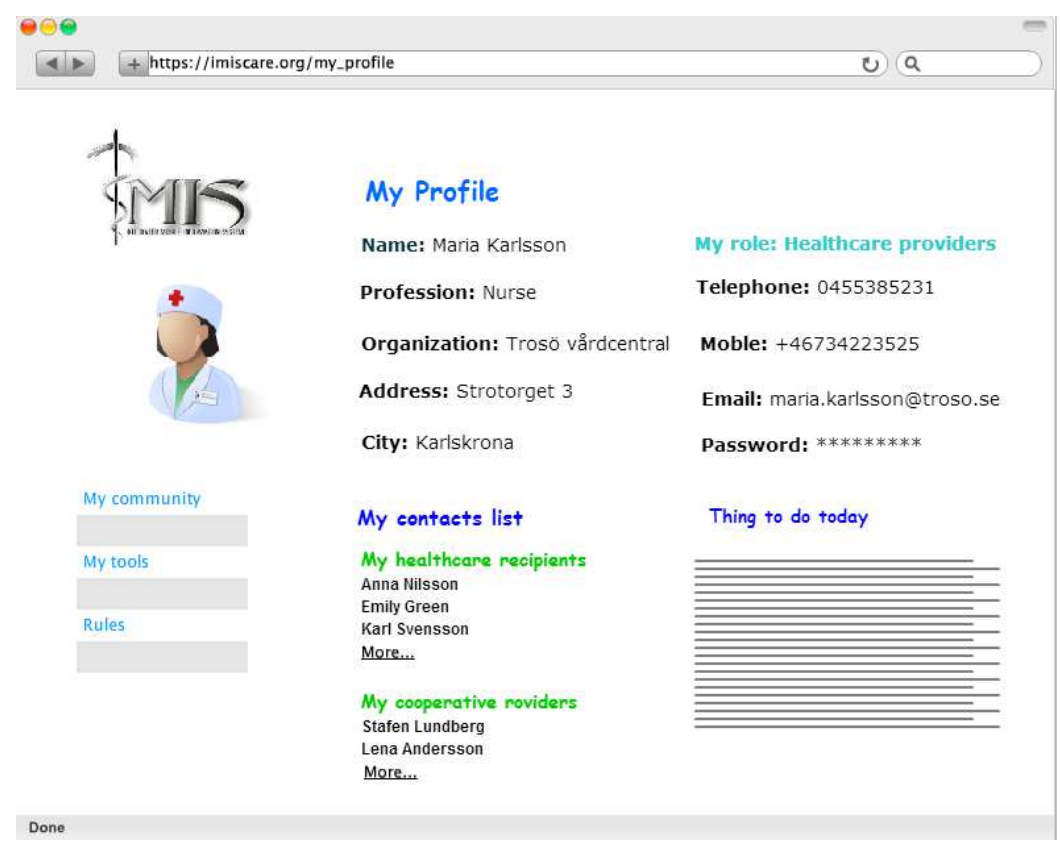

Fig. 6. Healthcare provider profile

The page "my community" is designed based on the needs of the respondents who answered the questionnaires see Fig.7. All the contact persons are listed in the right, including healthcare providers, family members and others. They can chat with each other when they are online, so the healthcare recipients can get feedback immediately from the healthcare providers without physically having to visit any healthcare organizations. The community also provides a space for users to post discussions, videos, links to websites etc. to share with their contacts. Another important function is that the community will always show information about the nearest healthcare centers based on the gathered user geographical data. The scheduled care activities will be shown rolling in red to remind the recipients. 


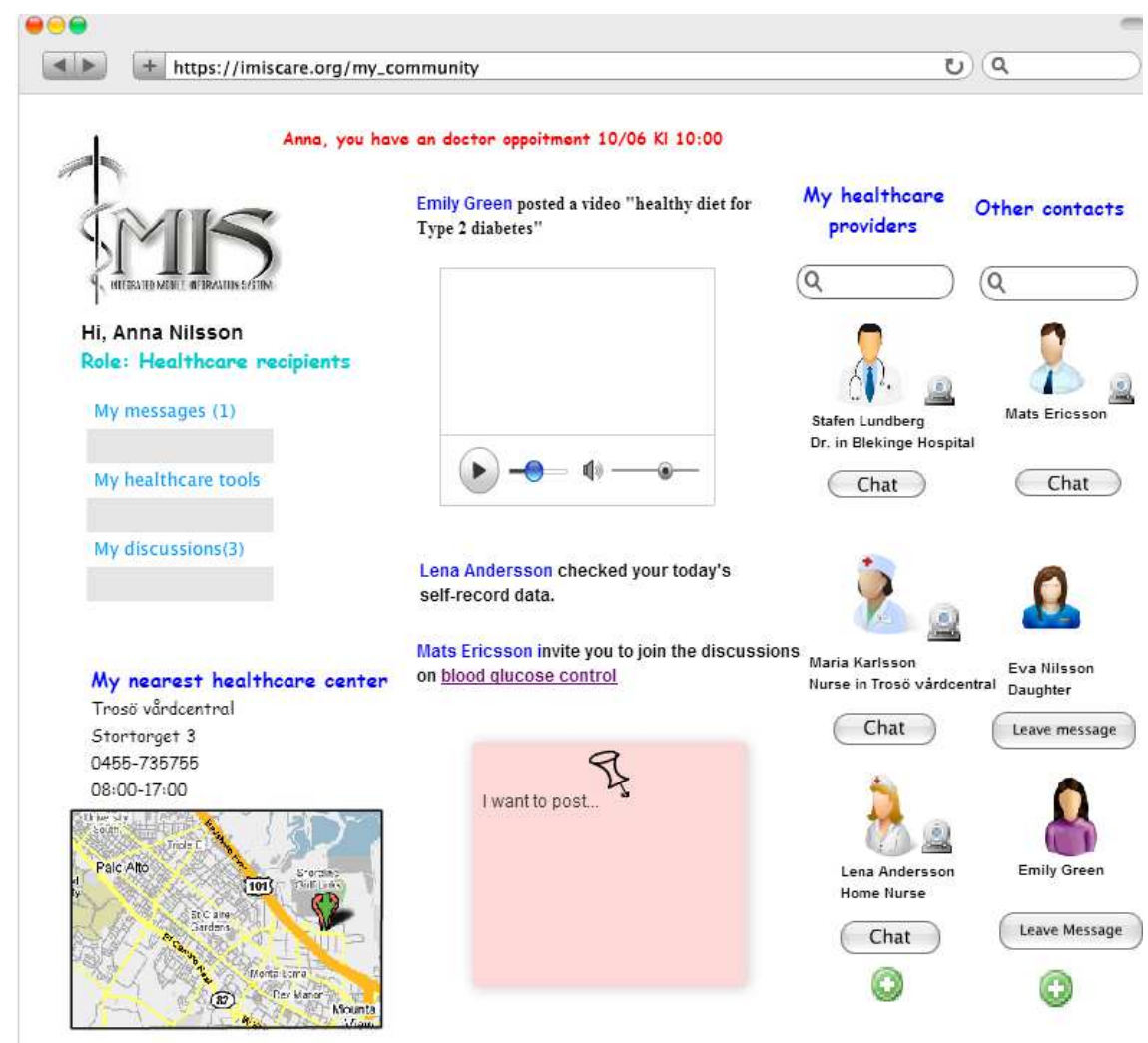

Fig. 7. Healthcare community page

The most important function of the home-based healthcare community is to provide a self-management platform for the healthcare recipients. In our design, it is in the recipients' "My tools" page, shown in Fig.8, the recipients update their basic physiological parameters, emotions and diet daily for record and trace. The authenticated healthcare providers can access the data and give some suggestions to recipients. The recipient can print out this data covering a long period as well to show the doctors and other care providers when they have an appointment. Online appointments are also proposed to reduce the waiting time for meeting doctors. In addition, personalized tools are introduced to the users so that they can use them based on their own needs. The healthcare providers' tools page includes functions for updating care history, appointment reminders, decision support systems for diagnoses and treatments and so on. 


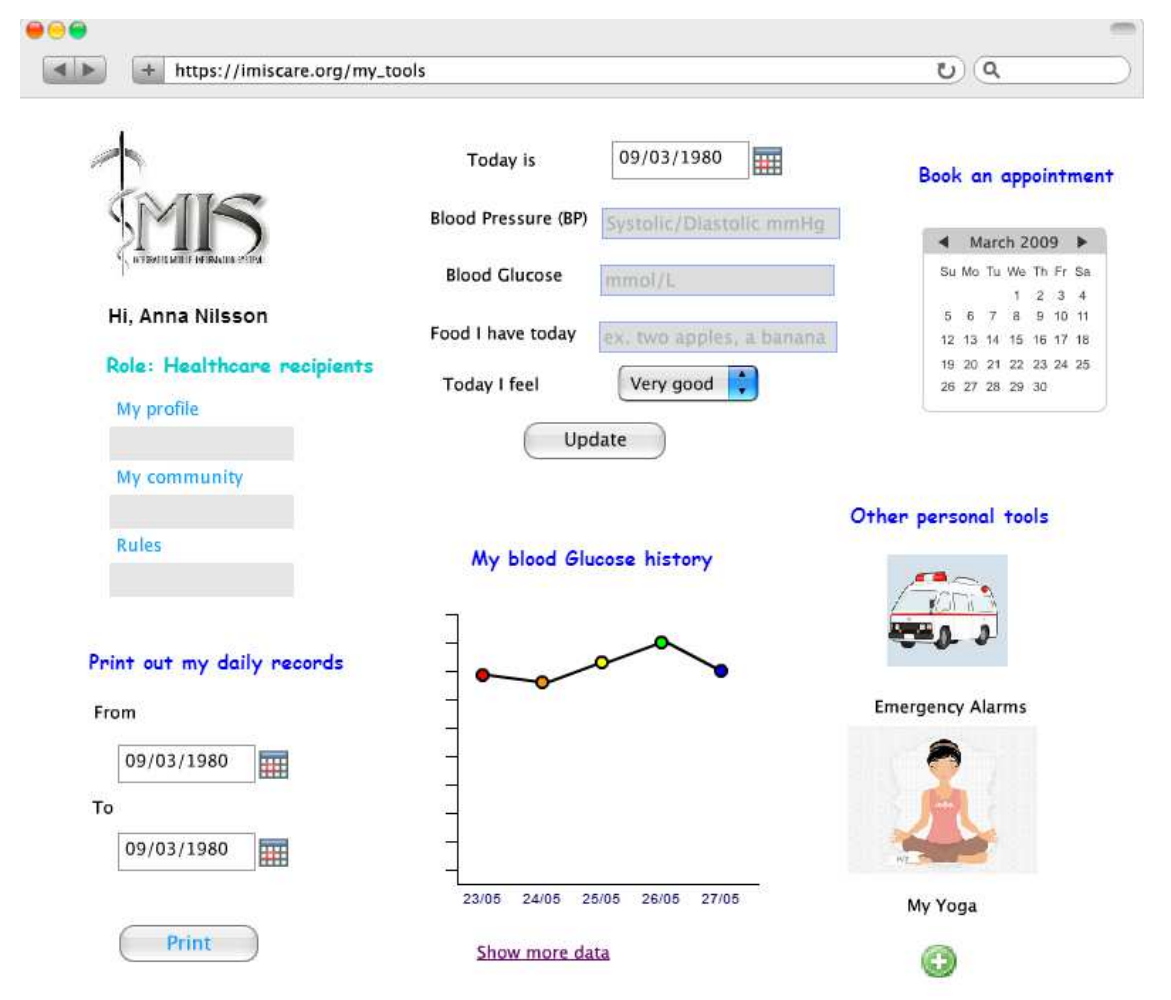

Fig. 8. Healthcare recipients' tool page

\section{$7 \quad$ Discussion and limitation}

The above prototype presents a general view of the design for a virtual community for home-based healthcare of chronic diseases. The design aimed to integrate healthcare recipients, healthcare providers and other relevant stakeholders into one community. This virtual community makes it possible to share the same platform for all the stakeholders who are engaged in the healthcare activity. With this shared community platform, the interoperability problems of current healthcare systems can be moderated. There exist various ways of applying ICT in eHealth, for example cloud computing. Cloud computing is beginning to demonstrate its great potentials in our daily healthcare due to its powerful services in managing big data, accessibility, flexibility, scalability and cost-effectiveness for services. Cloud technology mitigates the need to invest in IT infrastructure, by providing access to hardware, computing resources, applications, and services on a 'per use' model. And thus it dramatically brings down the cost and eases the adoption of technology. Besides, there are lots of existing privacy control mechanisms and security techniques in cloud computing which could help sensitive healthcare data protection. This will lead to radical new circumstances for offering eHealth services and constructing our new generation of healthcare information systems. The proposed virtual community in this paper will in 
the next step be implemented based on the cloud platform to ensure its accessibility, availability, and mobility, with careful design of security and privacy.

There are some limitations of this paper. Firstly, the healthcare recipient group that responded to our questionnaire was not large, due to the limited scope of our investigation and our restrictive rules (over 55 and have at least one chronic disease), so the care recipients' needs would require further investigation in the next step, based on a much larger sample. Secondly, as we are aiming to design a patient-centered healthcare community, the requirements of care providers would also require further investigation. In this case, they were gathered through interviews and informal discussions with only a few respondents, due to the time limitation for the study, so further work is needed to gain a better understanding of the needs of the care providers as well. However, the aim of this study was to develop a first prototype of a system for supporting the development of a virtual community for home-based chronic disease healthcare. This prototype can in future be used in a participatory design process to help future users envision and enact how new technological solutions can support and enhance healthy independent living and disease self-management.

\section{Conclusion and future work}

This paper proposed an online virtual community for home-based chronic disease healthcare. The design idea is inspired by social networking and based on the term "community" in activity theory. The requirements of the community were mainly gathered from the questionnaires with targeted healthcare recipients and interviewers with healthcare providers. We use "user stories" as they are used in agile software development to describe the requirements, and finally a prototype is designed based on the identified user requirements.

In the future, we will demonstrate this prototype to the potential users for evaluation. Further, more extensive interviews and surveys will be conducted with both healthcare recipients and healthcare providers to get more in-depth requirements from their respective perspectives. After this, we will develop the tools for supporting an online community based on cloud technology and test the proposed solution with future users for continued improvement and redesign. Ideally, such tools should be continuously further designed in use, together with the users, as part of quality lifecycle product and service management as well as part of the development of quality healthcare provision and self-management.

\section{References}

1. WHO $\mid$ Chronic diseases and health promotion, http://www.who.int/chp/en/.

2. What is Chronic Disease? | Center for Managing Chronic Disease - Putting People at the Center of Solutions, http://cmcd.sph.umich.edu/what-is-chronicdisease.html. 
3. Lin, C.-C., Lee, R.-G., Hsiao, C.-C.: A pervasive health monitoring service system based on ubiquitous network technology. Int. J. Med. Inf. 77, 461-469 (2008).

4. Chen, C.-M.: Web-based remote human pulse monitoring system with intelligent data analysis for home health care. Expert Syst. Appl. 38, 2011-2019 (2011).

5. Zimmer-Tamakoshi, L.: Sustainable Communities, Sustainable Development: Other Paths for Papua New Guinea. Pac. Aff. 86, 962-965 (2013).

6. Engeström, Y.: Learning by Expanding: An Activity-theoretical Approach to Developmental Research. Orienta-Konsultit Oy (1987).

7. Bai, G., Guo, Y.: A general architecture for developing a sustainable elderly care e-health system. In: 2011 8th International Conference on Service Systems and Service Management (ICSSSM). pp. 1-6 (2011).

8. HealthVault, https://www.healthvault.com/se/en.

9. Rogers, Y., Sharp, H., Preece, J.: Interaction Design: Beyond Human - Computer Interaction. John Wiley \& Sons (2011).

10.Patientjournalen - 1177 Vårdguiden - sjukdomar, undersökningar, hitta vård, etjänster, http://www.1177.se/Blekinge/Regler-ochrattigheter/Patientjournalen/\#section-3.

11.epSOS: About epSOS, http://www.epsos.eu/home/about-epsos.html.

12.1177 Vårdguiden - sjukdomar, undersökningar, hitta vård, e-tjänster, http://www.1177.se/Blekinge/.

13.Berenbach, B., Paulish, D., Kazmeier, J., Rudorfer, A.: Software \& Systems Requirements Engineering: In Practice. McGraw-Hill Osborne Media, New York, NY u.a. (2009).

14.Guide to Agile Practices, http://guide.agilealliance.org/guide/user-stories.html.

15. Glinz, M.: On Non-Functional Requirements. In: Requirements Engineering Conference, 2007. RE '07. 15th IEEE International. pp. 21-26 (2007).

16.Chung, L., Leite, J.C.S. do P.: On Non-Functional Requirements in Software Engineering. In: Borgida, A.T., Chaudhri, V.K., Giorgini, P., and Yu, E.S. (eds.) Conceptual Modeling: Foundations and Applications. pp. 363-379. Springer Berlin Heidelberg (2009).

17.ISO 9241-1:1992 - Ergonomic requirements for office work with visual display terminals (VDTs) -- $\quad$ Part 1: General introduction, http://www.iso.org/iso/iso_catalogue/catalogue_ics/catalogue_detail_ics.htm?csnu mber $=16873$.

18.Nielsen, J.: CHAPTER 1 - What Is Usability? User Exp. Re-Mastered. 3-22 (2010).

19. Nielsen, J.: Ten usability heuristics. Ten Usability Heuristics. (2005).

20. Braunstein, M.L.: Health informatics in the cloud. Springer (2012).

21.Goldstein, M.M., Rein, A.L., Heesters, M.M., Hughes, P.P., Williams, B., Weinstein, S.A.: Data segmentation in electronic health information exchange: Policy considerations and analysis. Data Segmentation Electron. Health Inf. Exch. Policy Consid. Anal. (2010).

22. Girija J. Narlikar, G.E.B.: Space-efficient scheduling of nested parallelism. ACM Trans. Program. Lang. Syst. 21, 138-173 (2002). 